\title{
DATA FUSION AND FILTERING VIA CALCULUS OF VARIATIONS
}

\author{
L. Fatone, ${ }^{1}$ P. Maponi, ${ }^{2}$ and F. Zirilli ${ }^{3}$ \\ ${ }^{1}$ Dipartimento di Matematica Pura ed Applicata, Università di Modena e Reggio Emilia, Via \\ Campi 213/b, 41100 Modena (MO), Italy, fatone.lorella@unimo.it, ${ }^{2},{ }^{2}$ Dipartimento di Matem- \\ atica e Informatica, Università di Camerino, Via Madonna delle Carceri, 62032 Camerino (MC), \\ Italy, pierluigi.maponi@unicam.it, ${ }^{3}$ Dipartimento di Matematica "G. Castelnuovo", Università \\ di Roma "La Sapienza", Piazzale Aldo Moro 2,00185 Roma, Italy, f.zirilli@caspurit
}

\begin{abstract}
We study the problem of urban areas detection from satellite images. In particular, we consider two types of satellite images: SAR (Synthetic Aperture Radar) images and optical images. We describe a simple algorithm for the detection of urban areas. We show that the performance of the detection algorithm can be improved using a fusion procedure of the SAR and optical images considered. The fusion algorithm presented in this paper is based on a simple use of ideas taken from calculus of variations and it makes possible to do together the filtering and the data fusion steps. Some numerical examples obtained processing real data are reported at the end of the paper. In the website http://web.unicam.it/ matinf/fatone/w1 several animations relative to these numerical examples can be seen.
\end{abstract}

keywords: Data fusion, Optimization algorithms, Urban areas detection

\section{Introduction}

The exploration of the Earth surface is an important use of remotely sensed data obtained from instruments on board of artificial satellites. These data can be used for many different purposes. In this paper we restrict our attention to the study of urban areas using SAR images and satellite optical images. Taking advantage of the physical properties of the electromagnetic waves with frequency in the range of SAR sensors and of optical sensors we adopt a well known urban areas detection algorithm based on the fact that urban areas can be recognized as the parts of the images containing the more brilliant pixels.

\footnotetext{
${ }^{*}$ The research on SAR/optical data fusion has been carried out with the support of ESA-ESRIN through the Esrin contract No. 13796/99/I-DC "Study of optical/SAR complementarity and data fusion techniques" granted to the Università di Camerino, Italy. "The authors thank A.R. Conn, N.I.M. Gould and Ph.L. Toint for making available, free of charge, the optimization package LANCELOT.
}

Please use the following format when citing this chapter:

Fatone, L., Maponi, P., and Zirilli, F., 2006, in IFIP International Federation for Information Processing, Volume 202, Systems, Control, Modeling and Optimization, eds. Ceragioli, F., Dontchev, A., Furuta, H., Marti, K., Pandolfi, L., (Boston: Springer), pp. 139-149. 
We can use the urban areas detection algorithm on SAR images and on optical images separately or, when the two images refer to the same scene, we can use jointly these two kinds of images in a fusion procedure to obtain new synthetic images, that is the fused images. The detection algorithm applied to the fused images should have a better performance than the one obtained on the original images considered separately. We show that this is really the case on some examples using real data. Moreover in this paper we propose a fusion procedure to combine the SAR images and the satellite optical images. Note that in order to perform meaningfully the fusion procedure the SAR and optical images to be fused must be not only relative to the same scene but also co-registered.

Many different authors have considered the fusion problem of SAR and optical images and the problem of urban areas detection, see, for example, [11], [6], [4], [7]. Image fusion is a special case of data fusion. For a general survey of this field see [1] and the references quoted there. The use of data fusion techniques is common practice in many fields different from remote sensing, such as inverse scattering, [5], and medical imaging, [8].

The mathematical formulation of the fusion procedure proposed here is based on a constrained optimization problem. This problem improves the mathematical formulation of the SAR/optical fusion procedure previously considered by the authors, see [4] and the web site http://web. unicam.it/matinf/fatone/ esrin.asp, where two different steps were performed: 1) segmentation and denoising of the images; 2) fusion of the images obtained from step 1). The fusion procedure considered in this paper performs these two steps together, through the formulation of a new optimization problem. This is obtained using a new objective function and adding some suitable constraints. The change made in the objective function is based on some simple ideas taken from calculus of variations. Mathematical models that simulate the functioning of the SAR and optical processors and describe the measuring processes are used in the constraints added to the optimization problem. These two facts correspond to a significant improvement of the work presented in [6], and [4]. The simple mathematical models of the SAR and optical sensors used to process the images considered are based on some classical results on remote sensing theory, see for example [9], [3], [10], and are given by integral relations between the measured data and the unknown measured quantities. We note that more sophisticated models of the SAR and the optical sensors can be integrated in the fusion procedure proposed. Moreover many different ad hoc detection algorithms can be used to characterize urban areas in the fused images.

In section 2 the mathematical models used to interpret the SAR and optical images are described. In section 3 we recall the urban areas detection algorithm presented in [4] and we present the improved version of the fusion procedure proposed here. In section 4 some implementation details of the algorithms presented in sections 2,3 and some numerical experiments are reported. In 
the website http : //web. unicam.it/matinf/fatone/w1 some animations relative to these numerical experiments can be seen.

\section{The mathematical models used to interpret SAR and optical images}

In this section we introduce two simple mathematical models to interpret satellite images with the purpose of showing how to integrate these models in the fusion procedure presented in Section 3.

Let us begin with the model for SAR images used here. We refer to the papers [9], [3] for a more detailed discussion. We denote with $\mathbf{R}$ the real line, and with $\mathbf{R}^{2}$ the two dimensional real Euclidean space. Let $A_{1}(\underline{\xi}), \underline{\xi}=\left(\xi_{1}, \xi_{2}\right)^{t} \in \mathbf{R}^{2}$ be the amplitude of the electromagnetic signal at the SAR frequency emitted from $\underline{\xi} \in \mathbf{R}^{2}$; the superscript $t$ means transposed. Note that in this model $A_{1}(\xi), \xi=\left(\xi_{1}, \xi_{2}\right)^{t} \in \mathbf{R}^{2}$ is a random variable. We have that in the position $\underline{x} \in \mathbf{R}^{2}$ the intensity SAR image $U_{1}(\underline{x})$ is given by:

$$
U_{1}(\underline{x})=\left(\mathbf{H}_{S}\left\langle A_{1}^{2}\right\rangle\right)(\underline{x})=\int_{\mathbf{R}^{2}}\left|h_{S}(\underline{\xi}-\underline{x})\right|^{2}\left\langle A_{1}^{2}(\underline{\xi})\right\rangle d \underline{\xi}
$$

where $\langle\cdot\rangle$ denotes the expected value of $\cdot$ and:

$$
\left|h_{S}(\underline{\xi})\right|^{2}=\frac{1}{\nu}\left[\frac{R_{1}}{\xi_{1}} \sin \left(\pi \frac{\xi_{1}}{R_{1}}\right) \frac{R_{2}}{\xi_{2}} \sin \left(\pi \frac{\xi_{2}}{R_{2}}\right)\right]^{2}, \underline{\xi} \in \mathbf{R}^{2},
$$

where $\nu>0$ is a normalization constant that makes the integral of $\left|h_{S}\right|^{2}$ over $\mathbf{R}^{2}$ equal to one, and $R_{1}, R_{2}>0$ are the resolutions of the SAR image along the two cartesian coordinates.

Let us consider now the model for optical images, see [10] for a more detailed discussion. We denote with $A_{2}(\xi)$ the amplitude of the electromagnetic signal at the optical frequency emitted from $\underline{\xi} \in \mathbf{R}^{2}$. This quantity is modeled as a random variable. Arguing as above we have that in the position $x \in \mathbf{R}^{2}$ the optical image $U_{2}(\underline{x})$ is given by:

$$
U_{2}(\underline{x})=\left(\mathbf{H}_{O}\left\langle A_{2}^{2}\right\rangle\right)(\underline{x})=\int_{\mathbf{R}^{2}}\left|h_{O}(\underline{\xi}-\underline{x})\right|^{2}\left\langle A_{2}^{2}(\underline{\xi})\right\rangle d \underline{\xi} .
$$

Let $F$ be the Fourier transform operator, $\underline{\omega} \in \mathbf{R}^{2}$ be the conjugate variable in the Fourier transform of $\underline{\xi} \in \mathbf{R}^{2}$. Note that $F\left(\left|h_{O}\right|^{2}\right)$ can be written as:

$$
F\left(\left|h_{O}\right|^{2}\right)(\underline{\omega})=T(\underline{\omega}) B(\underline{\omega}), \underline{\omega} \in \mathbf{R}^{2},
$$

where $T$ represents the functioning of the instrument and $B$ represents the perturbation due to the presence of the atmosphere; this perturbation is not negligible when modeling optical measurements. In [10] the following expressions 
of $T(\underline{\omega})$ and $B(\underline{\omega})$ are proposed:

$$
T(\underline{\omega})=\frac{1}{A} \int_{\mathbf{R}^{2}} Q_{0}(\lambda(\underline{\xi}+\underline{\omega})) Q_{0}(\lambda \underline{\xi}) d \underline{\xi}, B(\underline{\omega})=e^{-3.44\left(\frac{\lambda\|\underline{\omega}\|}{r_{0}}\right)^{5 / 3}}
$$

( $\underline{\omega}$ is in $\mathbf{R}^{2}$ ). $\lambda$ is the wavelength of the electromagnetic signal considered, $r_{0}>0$ is a parameter that controls the perturbation induced by the atmosphere on the image, $Q_{0}$ is the characteristic function of the disk with center the origin and with radius equal to the radius of the instrument lens, and $A$ is the area of the instrument lens. We note that the usual representation in pixels of the measured images that we denote with $u_{1}, u_{2}$ can be regarded as a piecewise constant approximation of the functions $U_{1}, U_{2}$ given by (1), (3). The value assumed by $u_{1}, u_{2}$ on a pixel can be understood as an "average" of $U_{1}, U_{2}$ respectively in the area corresponding to the pixel considered. These approximations are denoted with $u_{1}, u_{2}$ respectively. Moreover the support of the densities $\left\langle A_{1}^{2}\right\rangle,\left\langle A_{2}^{2}\right\rangle$ of the convolution equations (1), (3) is restricted to the support $R$ of the region covered by the measured images. In the sequel these restricted densities are denoted with $\chi_{1}, \chi_{2}$ respectively. Note that this restriction operation is justified since the convolution kernels appearing in equations (1), (3) have significant decay properties.

\section{The fusion procedure}

Let us describe the urban areas detection algorithm used here, see [4] for more details. Let $u$ be an image representing either a SAR image or an optical image or a synthetic image obtained from the fusion algorithm described in the sequel. The image $u$ by virtue of the usual pixel structure can be regarded as a piecewise constant real function defined on a rectangular region $R$ of the two-dimensional Euclidean space $\mathbf{R}^{2}$. We use the same notation $u$ to denote the image and the associated function, that is $u: R \rightarrow \mathbf{R}$. We note that the values of this function are the usual gray levels of the image. Let $\mu$ and $\sigma^{2}$ be respectively the mean and the variance of $u$. The urban areas detection algorithm used here consists of the following steps. Given a suitable positive integer $n$, the image $u$ is partitioned in non intersecting subimages having dimensions $n \times n$ pixels. Eventually we adjust the dimensions of the subimages lying on the right end side and on the upper end side of $u$ to fit with the dimensions of $u$. Let $L$ be the number of the subimages considered. For $l=1,2, \ldots, L$ let $\mu_{l}$ be the mean value associated to the gray levels of the $l$-th subimage. Since urban areas produce very brilliant texture (above all in SAR images) we assume that subimages containing urban areas are characterized by the fact of having a high value of the parameter:

$$
D_{l}=\left(\mu_{l}-\mu\right) / \sigma .
$$


Given a threshold $\Upsilon>0$, for $l=1,2, \ldots, L$ the $l$-th subimage is recognized as containing an urban area when:

$$
D_{l}>\Upsilon
$$

Let us describe the fusion procedures of SAR and optical images. In [4] (see also http://web.unicam.it/matinf/fatone/esrin.asp) we have proposed the following fusion procedure made of two steps: 1) denoising of $S A R$ and optical images; 2) fusion of the denoised images produced in 1).

Let $\hat{u}_{1}, \hat{u}_{2}$ be two images defined in the rectangular region $R$ representing the $\mathrm{SAR}$ and the optical image to be fused respectively. We assume that the images $\hat{u}_{1}, \hat{u}_{2}$ refer to the same scene, are coregistered, and that they have the same pixel structure. Step 1) consists in the solution of an initial boundary value problem, that is, for $i=1,2$, given the positive real numbers $a_{i}, T_{i}$, we compute the solution $v_{i}(\underline{x}, t), \underline{x} \in R, t \in\left[0, T_{i}\right]$, of the following problem:

$$
\begin{cases}\frac{\partial v_{i}}{\partial t}=\operatorname{div}\left(g_{a_{i}}\left(\left\|\nabla v_{i}\right\|\right) \nabla v_{i}\right), & (\underline{x}, t) \in R \times\left(0, T_{i}\right), \\ v_{i}(t=0)=\hat{u}_{i}, & \underline{x} \in R, \\ \frac{\partial v_{i}}{\partial \underline{n}}=0, & (\underline{x}, t) \in \partial R \times\left(0, T_{i}\right),\end{cases}
$$

where $\operatorname{div}(\cdot)$ and $\nabla(\cdot)$ are the divergence and the gradient of $\cdot$ with respect to $\underline{x}$ respectively, $\|\cdot\|$ is the Euclidean norm of $\cdot R$ is an open set, that we have assumed to be a rectangle, $\partial R$ is the boundary of the domain $R, \underline{n}(\underline{x})$ is the exterior unit normal vector to $\partial R$ in $\underline{x} \in \partial R, \frac{\partial}{\partial \underline{n}}$ is the usual directional derivative of - with respect to the direction $\underline{n}$, and finally $g_{a_{i}}(\eta)=\frac{1}{1+\eta^{2} / a_{i}^{2}}$, $\eta \in \mathbf{R}$, is the diffusion coefficient of problem (8). For $i=1,2$ and $0 \leq \tilde{t} \leq T_{i}$ we denote with $v_{i}(\tilde{t})$ the function $v_{i}(\underline{x}, \tilde{t}), \underline{x} \in R$. For later convenience we define $V_{1}=v_{1}\left(T_{1}\right), V_{2}=v_{2}\left(T_{2}\right)$. For $i=1,2, V_{i}$ is the denoised version of $\hat{u}_{i}$. Step 2) consists in the solution of an optimization problem. Let us consider a "structure" operator $S_{\tau_{1}, \tau_{2}}(\eta), \eta \in \mathbf{R}$, that is an approximation of the Heaviside function $H(\eta-\tau), \eta \in \mathbf{R}$, where $0<\tau_{1}<\tau<\tau_{2}$. For example $S_{\tau_{1}, \tau_{2}}(\eta)=H(\eta-\tau)$ for $\eta \in \mathbf{R} \backslash\left(\tau_{1}, \tau_{2}\right)$ and $S_{\tau_{1}, \tau_{2}}(\eta)$ is a twice continuously differentiable function for $\eta \in \mathbf{R}$ strictly increasing for $\eta \in\left[\tau_{1}, \tau_{2}\right]$. Given the denoised images $V_{1}, V_{2}$ and a suitable norm \|\|$\cdot \|$, the "fused" images $V_{1}^{*}, V_{2}^{*}$ are obtained as the minimizer of the following problem:

$$
\begin{aligned}
& \min _{w_{1}, w_{2}}\left\{\left\|S_{\tau_{1}, \tau_{2}}\left(\left\|\nabla w_{1}\right\|\right)-S_{\tau_{1}, \tau_{2}}\left(\left\|\nabla w_{2}\right\|\right)\right\|\left\|^{2}+\lambda_{1}\right\||| w_{1}-V_{1} \|^{2}\right. \\
& \left.+\lambda_{2}\left\|w_{2}-V_{2}\right\|^{2}\right\},
\end{aligned}
$$

where $\lambda_{1}, \lambda_{2}$ are suitable positive penalization parameters and $w_{1}, w_{2}$ are functions defined on $R$. We note that problem (9) is a fusion procedure, in fact problem (9) tries to change the variables $w_{1}, w_{2}$ in order to obtain structures $S_{\tau_{1}, \tau_{2}}\left(\left\|\nabla w_{1}\right\|\right), S_{\tau_{1}, \tau_{2}}\left(\left\|\nabla w_{2}\right\|\right)$ that are closer than $S_{\tau_{1}, \tau_{2}}\left(\left\|\nabla V_{1}\right\|\right)$ and 
$S_{\tau_{1}, \tau_{2}}\left(\left\|\nabla V_{2}\right\|\right)$, i.e. the initial structures, while the penalization terms force $w_{1}$ and $w_{2}$ to remain close to the denoised data $V_{1}$ and $V_{2}$ respectively.

Now we examine a new fusion procedure that unifies these two steps. Note that the denoising procedure (8) is well-suited for this purpose. More precisely we consider the following optimization problem: given $\epsilon>0, \hat{\lambda}_{1}>0, \hat{\lambda}_{2}>0$, the measured images $\hat{u}_{1}, \hat{u}_{2}$ and a suitable norm $\left\||\cdot \||\right.$, the fused densities $\chi_{1}^{*}$, $\chi_{2}^{*}$ are obtained as the minimizer of the following problem:

$$
\begin{aligned}
& \min _{\chi_{1}, \chi_{2} \geq 0}\left\{\left\|\mid S_{\tau_{1}, \tau_{2}}\left(\left\|\nabla \chi_{1}\right\|\right)-S_{\tau_{1}, \tau_{2}}\left(\left\|\nabla \chi_{2}\right\|\right)\right\| \|^{2}+J\left(\chi_{1}, \chi_{2}\right)\right\}, \\
& \text { subject to: } \hat{\lambda}_{1}\left\|\mathbf{H}_{S} \chi_{1}-\hat{u}_{1}\right\|+\hat{\lambda}_{2}\left\|\mathbf{H}_{O \chi_{2}}-\hat{u}_{2}\right\| \leq \epsilon,
\end{aligned}
$$

where $\mathbf{H}_{S}, \mathbf{H}_{O}$ are the integral operators defined in formulae (1), (3) respectively, $\chi_{1}, \chi_{2}$ are functions defined on $R$, the nonegativity constraints for $\chi_{1}$ and $\chi_{2}$ must be understood as pointwise inequalities and are dictated by elementary physics, and

$$
J\left(\chi_{1}, \chi_{2}\right)=l_{1} \int_{R} \ln \left(1+\frac{\left\|\nabla \chi_{1}(\underline{x})\right\|^{2}}{a_{1}^{2}}\right) d \underline{x}+l_{2} \int_{R} \ln \left(1+\frac{\left\|\nabla \chi_{2}(\underline{x})\right\|^{2}}{a_{2}^{2}}\right) d \underline{x},
$$

where $l_{1}, l_{2}$ are suitable positive parameters and $a_{1}, a_{2}$ are the parameters appearing in problem (8). From the solution $\chi_{1}^{*}, \chi_{2}^{*}$ of problem (10) the "fused" images $U_{1}^{*}, U_{2}^{*}$ are computed as follows:

$$
U_{1}^{*}(\underline{x})=\left(\mathbf{H}_{S} \chi_{1}^{*}\right)(\underline{x}), \underline{x} \in R, \quad U_{2}^{*}(\underline{x})=\left(\mathbf{H}_{O} \chi_{2}^{*}\right)(\underline{x}), \underline{x} \in R .
$$

Minimizing the objective function of problem (10) means to make small the quantity $\left\|\left|S_{\tau_{1}, \tau_{2}}\left(\left\|\nabla \chi_{1}\right\|\right)-S_{\tau_{1}, \tau_{2}}\left(\left\|\nabla \chi_{2}\right\|\right)\right|\right\|$, this corresponds to fuse the densities, $\chi_{1}, \chi_{2}$, and to make small $J\left(\chi_{1}, \chi_{2}\right)$, this corresponds to denoise the images following a procedure similar to the one defined in (8). In fact the trajectory $\left(v_{1}(\underline{x}, t), v_{2}(\underline{x}, t)\right), \underline{x} \in R$ defined for $t>0$ by (8) when we choose $\hat{u}_{1}, \hat{u}_{2}$, respectively as initial data is the steepest descent trajectory passing through $\left(\hat{u}_{1}, \hat{u}_{2}\right)$ associated to the minimization of the functional $J\left(\chi_{1}, \chi_{2}\right)$. More in detail let $v: R \rightarrow \mathbf{R}$ be a sufficiently regular function and let $f: \mathbf{R} \rightarrow$ $\mathbf{R}$ be a smooth function on $\mathbf{R}$. Let $\tilde{J}(v)=\int_{R} f(\|\nabla v(\underline{x})\|) d \underline{x}$ be a functional depending on the function $v$. Let $\Phi=\{\phi: R \rightarrow \mathbf{R}: \phi$ is a sufficiently regular function $\}$. From calculus of variations we know that the minimizers $v^{*}$ of $\tilde{J}$ must satisfy the first order necessary condition $\frac{\delta \tilde{J}}{\delta v}\left(v^{*}\right)=0$, where $\frac{\delta}{\delta v}$ means functional derivative of ', i.e. $\int_{R} f^{\prime}\left(\left\|\nabla v^{*}(\underline{x})\right\|\right) \frac{\nabla v^{*}(\underline{x}) \nabla \phi(\underline{x})}{\left\|\nabla v^{*}(\underline{x})\right\|} d \underline{x}=0$, $\forall \phi \in \Phi$, where $f^{\prime}$ denotes the derivative of $f$. Using the divergence theorem and assuming that $f^{\prime}(\eta) / \eta \neq 0, \eta \in \mathbf{R}$ we have:

$$
-\operatorname{div}\left(f^{\prime}\left(\left\|\nabla v^{*}(\underline{x})\right\|\right) \frac{\nabla v^{*}(\underline{x})}{\left\|\nabla v^{*}(\underline{x})\right\|}\right)=0, \underline{x} \in R, \quad \frac{\partial v^{*}(\underline{x})}{\partial \underline{n}(\underline{x})}=0, \underline{x} \in \partial R .
$$


Note that $v^{*}=$ constant satisfies (13) and comparing the boundary value problem (13) with problem (8) we can see that when $f^{\prime}(\eta) / \eta=g_{a_{i}}(\eta), \eta \in$ $\mathbf{R}, i=1,2$, problem (8) defines the steepest descent trajectory associated to the functional $\tilde{J}$ going through the point $\hat{u}_{i}, i=1,2$. We note that in this case the minimizer $v^{*}$ of $\tilde{J}$, obtained as steady state solution of problem (8), is $v_{i}^{*}=\int_{R} \hat{u}_{i}(\underline{x}) d \underline{x}, i=1,2$. From $f^{\prime}(\eta) / \eta=g_{a_{i}}(\eta), \eta \in \mathbf{R}$, we have: $f(\eta)=f_{i}(\eta)=a_{i}^{2} / 2 \ln \left(1+\eta^{2} / a_{i}^{2}\right)+c_{i}, \eta \in \mathbf{R}, i=1,2$, where $c_{i}, i=1,2$ are arbitrary constants that we choose equal zero.

We note that problem (10), (11), (1), (3) differs from problem (8), (9) mainly for two reasons: the use of the constraint corresponding to the mathematical models of the SAR and optical processors that replaces the penalization terms appearing in (9) and the new term $J$ in the objective function. Due to these differences problem (10), (11), (1), (3) can be seen as a refinement of problem (8), (9). In fact the use of the mathematical models (1), (3) that represent the measurement processes of the SAR and optical images makes problem (10), (11), (1), (3) more realistic than the problem considered previously. The parameters $l_{1}, l_{2}$ of problem (10) are tied to the parameters $T_{1}, T_{2}$ of problem (8) in the following sense: large values of $l_{1}, l_{2}$ correspond to large values of $T_{1}, T_{2}$, small values of $l_{1}, l_{2}$ correspond to small values of $T_{1}, T_{2}$. When the parameters $l_{1}, l_{2}$ take appropriate values minimizing the objective function of problem (10) corresponds both to solve problem (8) (due to the presence of the term $J$ ) and to make the fusion of the structures of $\chi_{1}$ and $\chi_{2}$ (due to the presence of the term $\left\|\left|S_{\tau_{1}, \tau_{2}}\left(\left\|\nabla \chi_{1}\right\|\right)-S_{\tau_{1}, \tau_{2}}\left(\left\|\nabla \chi_{2}\right\|\right) \|\right|^{2}\right)$. In fact this last term appears also in problem (9). That is solving problem (10) corresponds to performing step 1) (denoising) and 2) (fusion) of the fusion procedure used in [6] and in [4] together.

\section{The numerical experience}

We show some numerical results obtained using the fusion procedure and the urban areas detection algorithm proposed previously. These results are obtained processing real satellites data. In particular we consider two pairs of SAR/optical images, made of ERS SAR-average amplitude (range looks=azimuth looks=1) images and optical (one of the four SPOT-4 channels) images. These images have been provided to us by ESA-ESRIN, Frascati-Italy, with the authorization of SPOT Image. Each image is mono-channel, orthorectified using a Digital Elevation Model (DEM) of the observed scene. Moreover the SAR and optical images are coregistered. The first pair of images corresponds to a peri-urban area in the south of Paris, see Fig. 1 (a), (c). These images have $170 \times 180$ pixels and each pixel is $20 m \times 20 m$. The second pair corresponds to an area in the north of Paris that contains a part of the Roissy Charles de Gaulle airport, see Fig. 2 (a), (c). These images have $180 \times 180$ pixels and each pixel is 
$20 \mathrm{~m} \times 20 \mathrm{~m}$. The $20 \mathrm{~m} \times 20 \mathrm{~m}$ resolution of the images has been obtained preprocessing the original ERS and SPOT images. This pre-processing consists in resampling the images to have the same pixel of $20 \mathrm{~m} \times 20 \mathrm{~m}$ in both and coregistering the SAR and the optical images taking care of the DEM of the scene represented in the images. This pre-processing has been carried out by SPOT Image. We note that in these figures the white color represents high values of the pixel variable, i.e. gray level $=255$, and the black color represents low values of the pixel variable, i.e. gray level $=0$.
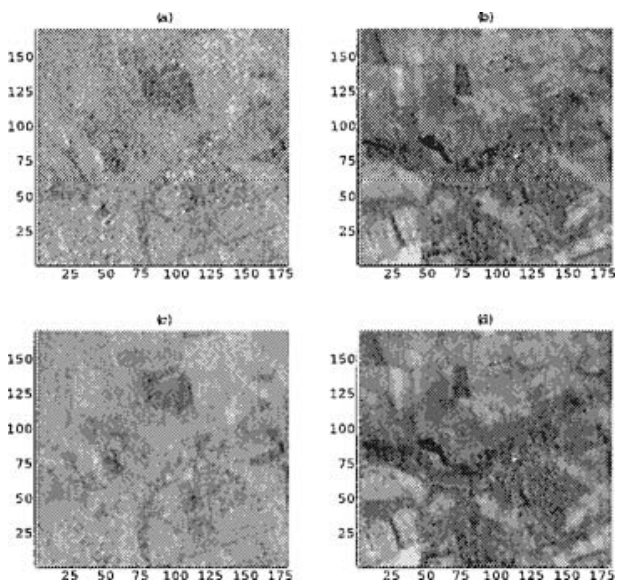

Figure 1. A peri-urban area south of Paris: (a) SAR image, (b) density corresponding to the SAR image obtained from the fusion procedure, (c) optical image, (d) density corresponding to the optical image obtained from the fusion procedure.

Problem (10) has been discretized using the natural pixels structure of the images and using the finite differences approximation of the derivatives and the rectangular quadrature formulae for the integrals appearing in (10). Moreover the finite dimensional optimization problem obtained in this way from problem (10) has been solved using the optimization software package LANCELOT (see [2]). Finally in the numerical solution of this optimization problem, due to the difficulties arising from the highly nonlinear function $S_{\tau_{1}, \tau_{2}}$, the fusion procedure is performed via an iterative process, where in each iteration is solved a problem of type (10) with a different choice of $\tau_{1}, \tau_{2}$ running from 1, 200 to $\tau_{1}^{*}, \tau_{2}^{*}$ where the values of $\tau_{1}^{*}, \tau_{2}^{*}$ are specified later. In the first optimization problem we use the images $\hat{u}_{1}, \hat{u}_{2}$, as initial guess for $\chi_{1}, \chi_{2}$, in the following optimization problems we use as initial guess the solution obtained in the previous optimization problem. Moreover in the constraints of problem (10), due to the large dimensions of the matrices obtained from the discretization of 

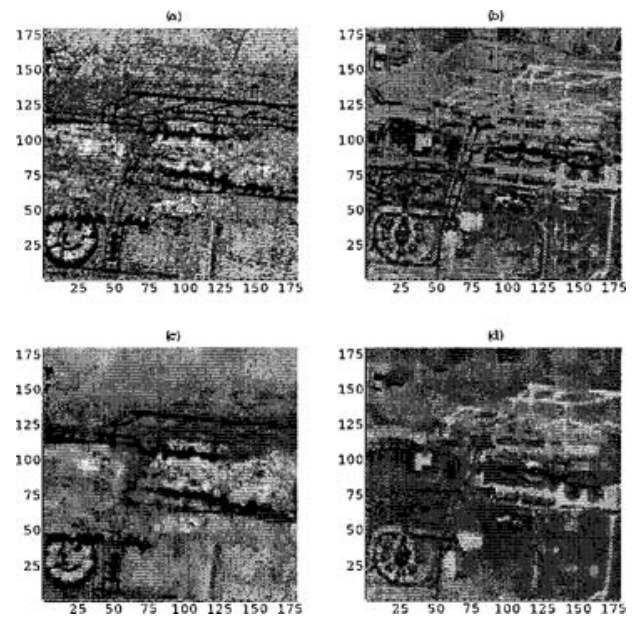

Figure 2. An area north of Paris containing a part of the Roissy Charles de Gaulle airport: (a) SAR image, (b) density corresponding to the SAR image obtained from the fusion procedure, (c) optical image, (d) density corresponding to the optical image obtained from the fusion procedure.

the integral operator $\mathbf{H}_{S}$ and $\mathbf{H}_{O}$, given a positive integer $\gamma$, we group together the pixels of the $\gamma \times \gamma$ subimages of the images considered. More precisely, each image is divided in non intersecting subimages having $\gamma \times \gamma$ pixels, and each subimage is considered as a new pixel in the constraints of problem (10). Moreover we define the gray level of this new pixel to be the average of the gray levels of the real pixels belonging to the $\gamma \times \gamma$ subimage. We note that in problem (10) the action of $\mathbf{H}_{S}$ on $\chi_{1}$ can be easily computed from formulae (1), (2), and that the action of $\mathbf{H}_{O}$ on $\chi_{2}$ can be easily computed from formulae (3), (4), (5). We note that the computation of the inverse Fourier transform of $T(\underline{\omega}), \underline{\omega} \in \mathbf{R}^{2}$ defined in (5) can be done explicitly in terms of the Bessel function of order one.

The numerical results of the fusion procedure shown in this section are obtained using the following values of the parameters mentioned previously: $R_{1}=R_{2}=20 \mathrm{~m}, A=0.28 \mathrm{~m}, r_{0}=\lambda^{6 / 5}, \lambda=5 \cdot 10^{-7} m, \tau_{1}^{*}=1, \tau_{2}^{*}=10$, $\hat{\lambda}_{1}=\hat{\lambda}_{2}=1, l_{1}=10^{-6}, l_{2}=10^{-7}, a_{1}=0.5, a_{2}=6, \gamma=10$. Finally the parameter $\epsilon$ is the upperbound for the weighted sum of the Euclidean norm of the remainders of the linear systems coming from the constraints appearing in (10) when we choose $\chi_{i}=\hat{u}_{i}, i=1,2$. Note that the (b), (d) parts of these figures do not show the same physical quantities than the (a), (c) parts of the figures. In fact in (a), (c) are reported the measured SAR and the measured optical images respectively, in (b), (d) are reported the densities $\chi_{1}^{*}, \chi_{2}^{*}$ solution 
of problem (10) that are related to the images via the mathematical models (1), (3). These densities are used in the urban areas detection algorithms. We note that the computational cost of the proposed fusion procedure is given by the computational cost of the optimization problem (10). More specifically the highly nonlinear character of the objective function and the huge number of independent variables coming from the discretized version of the unknowns of the problem make the solution of the nonlinear optimization problem a challenging task both from the mathematical and the computational point of view. We want to point out the improvement of the information content of the images consequence of the fusion procedure from the point of view of urban areas detection. Let $u$ be a generic image. We consider the parameters $D_{l}, l=1,2, \ldots$, $L$ defined in (6) associated to $u$. Moreover we denote $\bar{U}(u)$ the average of the parameters $D_{l}$ corresponding to the subimages of $u$ containing urban areas on the basis of the test (7) and we denote $\bar{V}(u)$ the average of the remaining parameters $D_{l}$, corresponding to subimages that, according to the test (7), do not correspond to urban areas. We introduce the following performance indices for $i=1,2$ see [4]:

$$
\alpha_{i}=\left(\bar{U}\left(\chi_{i}^{*}\right)-\bar{U}\left(\hat{u}_{i}\right)\right) / \bar{U}\left(\hat{u}_{i}\right), \beta_{i}=\left(\bar{V}\left(\chi_{i}^{*}\right)-\bar{V}\left(\hat{u}_{i}\right)\right) / \bar{V}\left(\hat{u}_{i}\right) .
$$

In section 3 we proposed to detect urban areas as the more brilliant areas in the image. So that it is easy to see that indices $\alpha_{1}, \alpha_{2}$ and $\beta_{1}, \beta_{2}$ give a measure of the improvement obtained by the fusion procedure in the urban areas detection algorithm and that positive values of the indices correspond to positive improvement. In the numerical experience that we report has been used $n=10, \Upsilon=0.25$ and we have obtained: $\alpha_{1}=0.35, \beta_{1}=0.51, \alpha_{2}=0.02$, $\beta_{2}=0.05$ for the first pair of images (see Fig. 1 ); $\alpha_{1}=0.23, \beta_{1}=0.38$, $\alpha_{2}=0.09, \beta_{2}=0.18$ for the second pair of images (see Fig. 2).

Finally we report another numerical experiment that shows the improvement obtained with the fusion procedure in the understanding of the scene contained in Fig. 2 (a), (c). In this experiment we compare the content of Fig. 2 (a), (c), the measured images, and the content of Fig. 2 (b), (d), the densities obtained from the fusion procedure, with a high resolution optical image of the same scene that we call ground truth (i.e.: an IRS-1C image). The IRS-1C image of the scene considered has pixels of size is $5 \mathrm{~m} \times 5 \mathrm{~m}$. We have coregistered manually the IRS-1C image with the images shown in Fig. 2 and we have computed the center of mass of several "objects" of size approximately 10 pixels ( 1 pixel $=20 \mathrm{~m} \times 20 \mathrm{~m})$ in the images of Fig. 2 and in the IRS-1C image. The centers of mass associated to the densities obtained from the fusion procedure appear to be more accurate than those computed from the measured images when compared with the centers of mass computed from the ground truth. The accuracy gained is of the order of a few meters (i.e. $5-10 \mathrm{~m}$ ). This experiment suggests that the fusion procedure introduced here can be an useful 
ingredient in a more ambitious procedure that includes for example automatic target recognition and location. Some animations relative to these numerical experiments can be seen in http://web. unicam.it/matinf/fatone/w1.

\section{References}

[1] Special issue on data fusion. Proceedings of the IEEE. 85:1-208, 1997.

[2] A.R. Conn, N.I.M. Gould, Ph.L. Toint. LANCELOT: A Fortran Package for Large Scale Nonlinear Optimization (Release A). Berlin, Springer-Verlag, 1992.

[3] M. Costantini, A. Farina, F. Zirilli. The fusion of different resolution SAR images. Proceedings of the IEEE. 85:139-146, 1997

[4] L. Fatone, P. Maponi, F. Zirilli. Fusion of SAR/Optical images to detect urban areas. Proceedings of the IEEE/ISPRS Joint Workshop on Remote Sensing and Data Fusion over Urban Areas, Roma Italy. 217-221, 2001.

[5] L. Fatone, P. Maponi, F. Zirilli. An image fusion approach to the numerical inversion of multifrequency electromagnetic scattering data. Inverse Problems. 17:1689-1702, 2001.

[6] L. Fatone, P. Maponi, F. Zirilli. Data fusion and nonlinear optimization. SIAM News. 35(1):4;10, 2002.

[7] C. Gouinaud, F. Tupin, H. Maître. Potential and use of radar images for characterization and detection of urban areas. IGARSS'96. Lincoln, Nebraska, USA. 1:474-476, 1996.

[8] K. Kneöaurek, M. Ivanovic, J. Machac, D.A. Weber. Medical image registration. Europhysics News. 31:5-8, 2000.

[9] C.J. Oliver. Information from SAR Images. Journal of Physics D-Applied Physics. 24:1493-1514, 1991.

[10] F. Roddier. The effects of atmospheric turbulence in optical astronomy. In Progress in Optics, North-Holland. Wolf, E. (Ed.) 19:281-376, 1981.

[11] A.H. Soldberg. Multisource classification of remotely sensed data: fusion of Landsat TM and SAR images. IEEE Transactions on Geoscience and Remote Sensing. 32:768-776, 1994. 